\title{
BALANCED HKT METRICS AND STRONG HKT METRICS ON HYPERCOMPLEX MANIFOLDS
}

\author{
Misha VERBitsky
}

\begin{abstract}
A manifold $(M, I, J, K)$ is called hypercomplex if $I, J, K$ are complex structures satisfying quaternionic relations. A quaternionic Hermitian hypercomplex manifold is called HKT (hyperkähler with torsion) if the $(2,0)$-form $\Omega$ associated with the corresponding $S p(n)$-structure satisfies $\partial \Omega=0$. A Hermitian metric $\omega$ on a complex manifold is called balanced if $d^{*} \omega=0$. We show that balanced HKT metrics are precisely the quaternionic Calabi-Yau metrics defined in terms of the quaternionic Monge-Ampère equation. In particular, a balanced HKT-metric is unique in its cohomology class, and it always exists if the quaternionic Calabi-Yau theorem is true. We investigate the cohomological properties of strong HKT metrics (the quaternionic Hermitian metrics, satisfying, in addition to the HKT condition, the relation $d d^{c} \omega=0$ ), and show that the space of strong HKT metrics is finite-dimensional. Using Howe's duality for representations of $S p(n)$, we prove a hyperkähler version of Hodge-Riemann bilinear relations. We use it to show that a manifold admitting a balanced HKT-metric never admits a strong HKT-metric, if $\operatorname{dim}_{\mathbb{R}} M \geqslant 12$.
\end{abstract}

\section{Introduction}

The notion of an HKT manifold was introduced by the physicists, but it proved to be immensely useful in matematics.

A hypercomplex manifold is a manifold equipped with almost complex structure operators $I, J, K: T M \longrightarrow T M$, integrable and satisfying the standard quaternionic relations $I^{2}=J^{2}=K^{2}=I J K=-\operatorname{Id}_{T M}$.

This gives a quaternionic algebra action on $T M$; the group $S p(1) \cong S U(2)$ of unitary quaternions acts on all tensor powers of $T M$ by multiplicativity.

A quaternionic Hermitian structure on a hypercomplex manifold is an $S U(2)$ invariant Riemannian metric. Such a metric gives a reduction of the structure group of $M$ to $S p(n)=U(n, \mathbb{H})$.

With any quaternionic Hermitian structure on $M$ one associates a non-degenerate $(2,0)$-form $\Omega \in \Lambda_{I}^{2,0}(M)$, as follows. ${ }^{1}$ Consider the differential forms

$$
\omega_{I}(\cdot, \cdot):=g(\cdot, I \cdot), \quad \omega_{J}(\cdot, \cdot):=g(\cdot, J \cdot), \quad \omega_{K}(\cdot, \cdot):=g(\cdot, K \cdot) .
$$

It is easy to check that the form $\Omega:=\omega_{J}+\sqrt{-1} \Omega_{K}$ is of Hodge type $(2,0)$ with respect to $I$.

Received by the editors September 1, 2008.

Misha Verbitsky is supported by CRDF grant RM1-2354-MO02.

${ }^{1} \Lambda^{*}(M)$ denotes the bundle of differential forms, and $\Lambda^{*}(M)=\oplus_{p, q} \Lambda_{I}^{p, q}(M)$ its Hodge decomposition, taken with respect to the complex structure $I$ on $M$. 
If the form $\Omega$ is closed, one has $d \omega_{I}=d \omega_{J}=d \omega_{K}=0$, and the manifold $(M, I, J, K, g)$ is called hyperkähler ([Bes]). The hyperkähler condition is very restrictive.

A hypercomplex, quaternionic Hermitian manifold $(M, I, J, K, g)$ is called an HKT-manifold (hyperkähler with torsion) if $\partial \Omega=0$, where $\partial$ denotes the $(1,0)$-part of the differential. In other words, a manifold is HKT if $d \Omega \in \Lambda_{I}^{2,1}(M)$.

The form $\Omega \in \Lambda_{I}^{2,0}(M)$ is called an HKT-form on $(M, I, J, K)$.

Remark 1.1: The quaternionic Hermitian form $g$ can be easily reconstructed from $\Omega$. Indeed, for any $x, y \in T_{I}^{1,0}(M)$, one has

$$
2 g(x, \bar{y})=\Omega(x, J(\bar{y})),
$$

as a trivial calculation implies.

HKT-manifolds were first introduced by the physicists ([HP]; see also [GP]) in a completely different context. Given a complex Hermitian manifold $(M, I, g)$, one defines a Bismut connection $\nabla: T M \longrightarrow T M \otimes \Lambda^{1} M$, determined by the following properties

(i): $\nabla I=\nabla g=0$

(ii): The torsion form $T_{\nabla} \in T M \otimes \Lambda^{2} M$ is totally antisymmetric, if one identifies $T M \otimes \Lambda^{2} M$ with $\Lambda^{1} M \otimes \Lambda^{2} M$.

This connection has its origins also in physics, due to A. Strominger ([SSTV]), who defined it earlier than Bismut's paper appeared. It is well known such $\nabla$ exists, and it is unique (see e.g. [G]). The torsion 3 -form $T$ of Bismut connection can be written down explicitly in terms of its Hermitian form $\omega$ :

$$
T=-I d \omega .
$$

Now, suppose that $(M, I, J, K, g)$ is a hypercomplex, quaternionic Hermitian manifold. The metric $g$ can be used to define the Bismut connections $\nabla_{I}, \nabla_{J}, \nabla_{K}$ associated with $I, J, K$. It is known (see e.g. [GP]) that $\nabla_{I}=\nabla_{J}=\nabla_{K}$ if and only if $(M, I, J, K, g)$ is HKT. This was the original definition of HKT structures ([HP]).

Remark 1.2: Let $(M, g)$ be a Riemannian manifold, and $\nabla$ a connection on $M$ which satisfies $\nabla g=0$. Such a connection is uniquely determined by its torsion form; this is proven by the same argument as used to show existence and uniqueness of the LeviCivita connection. However, the torsion term of the Bismut connection is written as $T=-I d \omega$. Therefore, $\nabla_{I}=\nabla_{J}=\nabla_{K}$ is equivalent to the following relation:

$$
-I d \omega_{I}=-J d \omega_{J}=-K d \omega_{K}
$$

This relation can be used as one more definition of HKT structures.

\section{Quaternionic Dolbeault complex on a hypercomplex manifold}

2.1. Quaternionic Dolbeault complex: a definition. It is well-known that any irreducible representation of $S U(2)$ over $\mathbb{C}$ can be obtained as a symmetric power $S^{i}\left(V_{1}\right)$, where $V_{1}$ is a fundamental 2-dimensional representation. We say that a representation $W$ has weight $i$ if it is isomorphic to $S^{i}\left(V_{1}\right)$. A representation is said to be pure of weight $i$ if all its irreducible components have weight $i$. 
Remark 2.1: The Clebsch-Gordan formula (see [Hu]) claims that the weight is multiplicative, in the following sense: if $i \leqslant j$, then

$$
V_{i} \otimes V_{j}=\bigoplus_{k=0}^{i} V_{i+j-2 k},
$$

where $V_{i}=S^{i}\left(V_{1}\right)$ denotes the irreducible representation of weight $i$.

Let $M$ be a hypercomplex manifold, $\operatorname{dim}_{\mathbb{H}} M=n$. There is a natural multiplicative action of $S U(2) \subset \mathbb{H}^{*}$ on $\Lambda^{*}(M)$, associated with the hypercomplex structure.

Let $V^{i} \subset \Lambda^{i}(M)$ be a maximal $S U(2)$-invariant subspace of weight $<i$. The space $V^{i}$ is well defined, because it is a sum of all irreducible representations $W \subset \Lambda^{i}(M)$ of weight $<i$. Since the weight is multiplicative (Remark 2.1), $V^{*}=\bigoplus_{i} V^{i}$ is an ideal in $\Lambda^{*}(M)$.

It is easy to see that the de Rham differential $d$ increases the weight by 1 at most. Therefore, $d V^{i} \subset V^{i+1}$, and $V^{*} \subset \Lambda^{*}(M)$ is a differential ideal in the de Rham DG-algebra $\left(\Lambda^{*}(M), d\right)$.

Definition 2.2: Denote by $\left(\Lambda_{+}^{*}(M), d_{+}\right)$the quotient algebra $\Lambda^{*}(M) / V^{*}$ It is called the quaternionic Dolbeault algebra of $M$, or the quaternionic Dolbeault complex (qD-algebra or $\mathrm{qD}$-complex for short).

Remark 2.3: The complex $\left(\Lambda_{+}^{*}(M), d_{+}\right)$was constructed much earlier by Capria and Salamon, ([CS]) in a different (and much more general) situation, and much studied since then.

\subsection{The Hodge decomposition of the quaternionic Dolbeault complex. .}

The Hodge bigrading is compatible with the weight decomposition of $\Lambda^{*}(M)$, and gives a Hodge decomposition of $\Lambda_{+}^{*}(M)([\mathrm{V} 3])$ :

$$
\Lambda_{+}^{i}(M)=\bigoplus_{p+q=i} \Lambda_{+, I}^{p, q}(M) .
$$

The spaces $\Lambda_{+, I}^{p, q}(M)$ are the weight spaces for a particular choice of a Cartan subalgebra in $\mathfrak{s u}(2)$. The $\mathfrak{s u}(2)$-action induces an isomorphism of the weight spaces within an irreducible representation. This gives the following result.

Proposition 2.4: Let $(M, I, J, K)$ be a hypercomplex manifold and

$$
\Lambda_{+}^{i}(M)=\bigoplus_{p+q=i} \Lambda_{+, I}^{p, q}(M)
$$

the Hodge decomposition of $\mathrm{qD}$-complex defined above. Then there is a natural isomorphism

$$
\Lambda_{+, I}^{p, q}(M) \cong \Lambda^{p+q, 0}(M, I) .
$$

Proof: See [V3]. 
This isomorphism is compatible with a natural algebraic structure on

$$
\bigoplus_{p+q=i} \Lambda^{p+q, 0}(M, I),
$$

and with the Dolbeault differentials, in the following way.

Let $(M, I, J, K)$ be a hypercomplex manifold. We extend

$$
J: \Lambda^{1}(M) \longrightarrow \Lambda^{1}(M)
$$

to $\Lambda^{*}(M)$ by multiplicativity. Recall that

$$
J\left(\Lambda^{p, q}(M, I)\right)=\Lambda^{q, p}(M, I),
$$

because $I$ and $J$ anticommute on $\Lambda^{1}(M)$. Denote by

$$
\partial_{J}: \Lambda^{p, q}(M, I) \longrightarrow \Lambda^{p+1, q}(M, I)
$$

the operator $J \circ \bar{\partial} \circ J$, where $\bar{\partial}: \Lambda^{p, q}(M, I) \longrightarrow \Lambda^{p, q+1}(M, I)$ is the standard Dolbeault operator on $(M, I)$, that is, the (0.1)-part of the de Rham differential. Since $\bar{\partial}^{2}=0$, we have $\partial_{J}^{2}=0$. In [V3] it was shown that $\partial$ and $\partial_{J}$ anticommute:

$$
\left\{\partial_{J}, \partial\right\}=0 .
$$

Consider the quaternionic Dolbeault complex $\left(\Lambda_{+}^{*}(M), d_{+}\right)$constructed in Subsection 2.1. Using the Hodge bigrading, we can decompose this complex, obtaining a bicomplex

$$
\Lambda_{+, I}^{*, *}(M) \stackrel{d_{+, I}^{1,0}, d_{+, I}^{0,1}}{\longrightarrow} \Lambda_{+, I}^{*, *}(M)
$$

where $d_{+, I}^{1,0}, d_{+, I}^{0,1}$ are the Hodge components of the quaternionic Dolbeault differential $d_{+}$, taken with respect to $I$.

Theorem 2.5: Under the multiplicative isomorphism

$$
\Lambda_{+, I}^{p, q}(M) \cong \Lambda^{p+q, 0}(M, I)
$$

constructed in Proposition 2.4, $d_{+}^{1,0}$ corresponds to $\partial$ and $d_{+}^{0,1}$ to $\partial_{J}$ :

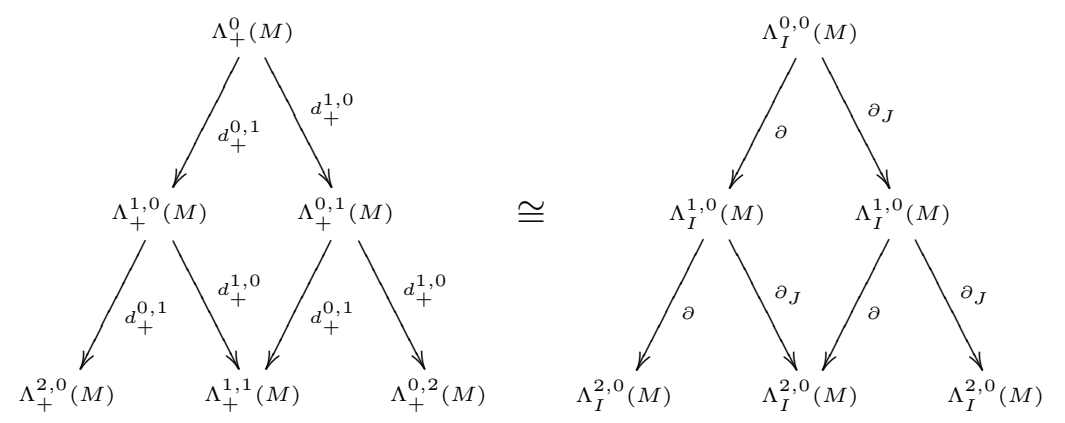

Moreover, under this isomorphism, $\omega_{I} \in \Lambda_{+, I}^{1,1}(M)$ corresponds to $\Omega \in \Lambda_{I}^{2,0}(M)$.

Proof: See [V3] or [V7]. 
2.3. Positive $(2,0)$-forms on hypercomplex manifolds. The notion of positive $(2 p, 0)$-forms on hypercomplex manifolds (sometimes called q-positive, or $\mathbb{H}$-positive) was developed in [V4] and [AV1] (see also [AV2] and [V8]). For our present purposes, only $(2,0)$-forms are interesting, but everything can be immediately generalized to a general situation

Let $\eta \in \Lambda_{I}^{p, q}(M)$ be a differential form. Since $I$ and $J$ anticommute, $J(\eta)$ lies in $\Lambda_{I}^{q, p}(M)$. Clearly, $\left.J^{2}\right|_{\Lambda_{I}^{p, q}(M)}=(-1)^{p+q}$. For $p+q$ even, $\left.J\right|_{\Lambda_{I}^{p, q}(M)}$ is an anticomplex involution, that is, a real structure on $\Lambda_{I}^{p, q}(M)$. A form $\eta \in \Lambda_{I}^{2 p, 0}(M)$ is called real if $J(\bar{\eta})=\eta$.

For a real $(2,0)$-form $\eta$,

$$
\eta(x, J(\bar{x})))=\bar{\eta}\left(J(x), J^{2}(\bar{x})\right)=\bar{\eta}(\bar{x}, J(x)),
$$

for any $x \in T_{I}^{1,0}(M)$. From a definition of a real form, we obtain that the scalar $\eta(x, J(\bar{x}))$ is always real.

Definition 2.6: A real $(2,0)$-form $\eta$ on a hypercomplex manifold is called positive if $\eta(x, J(\bar{x})) \geqslant 0$ for any $x \in T_{I}^{1,0}(M)$, and strictly positive if this inequality is strict, for all $x \neq 0$.

An HKT-form $\Omega \in \Lambda_{I}^{2,0}(M)$ of any HKT-structure is strictly positive, as follows from Remark 1.1. Moreover, HKT-structures on a hypercomplex manifold are in one-to-one correspondence with closed, strictly positive $(2,0)$-forms.

The analogy between Kähler forms and HKT-forms can be pushed further; it turns out that any HKT-form $\Omega \in \Lambda_{I}^{2,0}(M)$ has a local potential $\varphi \in C^{\infty}(M)$, in such a way that $\partial \partial_{J} \varphi=\Omega([\operatorname{AV} 1])$. Here $\partial \partial_{J}$ is a composition of $\partial$ and $\partial_{J}$ defined on quaternionic Dolbeault complex as above (these operators anticommute).

\section{3. $S L(n, \mathbb{H})$-manifolds}

3.1. An introduction to $S L(n, \mathbb{H})$-geometry. As Obata has shown $([\mathrm{Ob}])$, a hypercomplex manifold $(M, I, J, K)$ admits a necessarily unique torsion-free connection, preserving $I, J, K$. The converse is also true: if a manifold $M$ equipped with an action of $\mathbb{H}$ admits a torsion-free connection preserving the quaternionic action, it is hypercomplex. This implies that a hypercomplex structure on a manifold can be defined as a torsion-free connection with holonomy in $G L(n, \mathbb{H})$. This connection is called the Obata connection on a hypercomplex manifold.

Connections with restricted holonomy are one of the central notions in Riemannian geometry, due to Berger's classification of irreducible holonomy of Riemannian manifolds. However, a similar classification exists for a general torsion-free connection ([MS]). In the Merkulov-Schwachhöfer list, only three subroups of $G L(n, \mathbb{H})$ occur. In addition to the compact group $S p(n)$ (which defines hyperkähler geometry), also $G L(n, \mathbb{H})$ and its commutator $S L(n, \mathbb{H})$ appear, corresponding to hypercomplex manifolds and hypercomplex manifolds with trivial determinant bundle, respectively. Both of these geometries are interesting, rich in structure and examples, and deserve detailed study. 
It is easy to see that $(M, I)$ has holomorphically trivial canonical bundle, for any $S L(n, \mathbb{H})$-manifold $(M, I, J, K)([\mathrm{V} 6])$. For a hypercomplex manifold with trivial canonical bundle admitting an HKT metric, a version of Hodge theory was constructed ([V3]). Using this result, it was shown that a compact hypercomplex manifold with trivial canonical bundle has holonomy in $S L(n, \mathbb{H})$, if it admits an HKT-structure ([V6]).

In [BDV], it was shown that holonomy of all hypercomplex nilmanifolds lies in $S L(n, \mathbb{H})$. Many (probably, most) working examples of hypercomplex manifolds are in fact nilmanifolds, and by this result they all belong to the class of $S L(n, \mathbb{H})$-manifolds.

The $S L(n, \mathbb{H})$-manifolds were studied in [AV2] and [V8], because on such manifolds the quaternionic Dolbeault complex is identified with a part of de Rham complex (Proposition 3.1). Under this identification, $\mathbb{H}$-positive forms become positive in the usual sense, and $\partial, \partial_{J}$-closed or exact forms become $\partial, \bar{\partial}$-closed or exact. This linearalgebraic identification is especially useful in the study of quaternionic Monge-Ampère equation (Subsection 4.1).

3.2. The map $\mathcal{V}_{p, q}: \Lambda_{I}^{p+q, 0}(M) \longrightarrow \Lambda_{I}^{n+p, n+q}(M)$ on $S L(n, \mathbb{H})$-manifolds. Let $(M, I, J, K)$ be an $S L(n, \mathbb{H})$-manifold, $\operatorname{dim}_{\mathbb{R}} M=4 n$, and

$$
\mathcal{R}_{p, q}: \Lambda_{I}^{p+q, 0}(M) \longrightarrow \Lambda_{I,+}^{p, q}(M)
$$

the isomorphism induced by $\mathfrak{s u}(2)$-action as in Theorem 2.5 . Consider the projection

$$
\Lambda_{I}^{p, q}(M) \longrightarrow \Lambda_{I,+}^{p, q}(M),
$$

and let $R: \Lambda_{I}^{p, q}(M) \longrightarrow \Lambda_{I}^{p+q, 0}(M)$ denote the composition of (3.1) and $\mathcal{R}_{p, q}^{-1}$.

Let $\Phi_{I}$ be a nowhere degenerate holomorphic section of $\Lambda_{I}^{2 n, 0}(M)$. Assume that $\Phi_{I}$ is real, that is, $J\left(\Phi_{I}\right)=\bar{\Phi}_{I}$, and positive. Existence of such a form is equivalent to $\operatorname{Hol}(M) \subset S L(n, \mathbb{H})$ (Lemma 4.3). It is often convenient to define $S L(n, \mathbb{H})$-structure by fixing the quaternionic action and the holomorphic form $\Phi_{I}$.

Define the map

$$
\mathcal{V}_{p, q}: \Lambda_{I}^{p+q, 0}(M) \longrightarrow \Lambda_{I}^{n+p, n+q}(M)
$$

by the relation

$$
\mathcal{V}_{p, q}(\eta) \wedge \alpha=\eta \wedge R(\alpha) \wedge \bar{\Phi}_{I}
$$

for any test form $\alpha \in \Lambda_{I}^{n-p, n-q}(M)$.

The map $\mathcal{V}_{p, p}$ is especially remarkable, because it maps closed, positive $(2 p, 0)$ forms to closed, positive $(n+p, n+p)$-forms, as the following proposition implies.

Proposition 3.1: Let $\left(M, I, J, K, \Phi_{I}\right)$ be an $S L(n, \mathbb{H})$-manifold, and

$$
\mathcal{V}_{p, q}: \Lambda_{I}^{p+q, 0}(M) \longrightarrow \Lambda_{I}^{4 n-p, 4 n-q}(M)
$$

the map defined above. Then

(i): $\mathcal{V}_{p, q}(\eta)=\mathcal{R}_{p, q}(\eta) \wedge \mathcal{V}_{0,0}(1)$

(ii): The map $\mathcal{V}_{p, q}$ is injective, for all $p, q$.

(iii): $(\sqrt{-1})^{(n-p)^{2}} \mathcal{V}_{p, p}(\eta)$ is real if and only $\eta \in \Lambda_{I}^{2 p, 0}(M)$ is real, and weakly positive if and only if $\eta$ is weakly positive. 
(iv): $\mathcal{V}_{p, q}(\partial \eta)=\partial \mathcal{V}_{p-1, q}(\eta)$, and $\mathcal{V}_{p, q}\left(\partial_{J} \eta\right)=\bar{\partial} \mathcal{V}_{p, q-1}(\eta)$

(v): $\mathcal{V}_{0,0}(1)=\lambda \mathcal{R}_{n, n}\left(\Phi_{I}\right)$, where $\lambda$ is a positive rational number, depending only on the dimension $n$.

Proof: See [V8], Proposition 4.2, or [AV2], Theorem 3.6.

3.3. Algebra generated by $\omega_{I}, \omega_{J}, \omega_{K}$. Let $(M, I, J, K, g)$ be a quaternionic Hermitian manifold. Consider the algebra $A^{*}=\oplus A^{2 i}$ generated by $\omega_{I}, \omega_{J}$, and $\omega_{K}$. In [V1], this algebra was computed explicitly. It was shown that, up to the middle degree, $A^{*}$ is a symmetric algebra with generators $\omega_{I}, \omega_{J}, \omega_{K}$. The algebra $A^{*}$ has Hodge bigrading $A^{k}=\bigoplus_{p+q=k} A^{p, q}$. From the Clebsch-Gordan formula, we obtain that $A_{+}^{2 i}:=\Lambda_{+}^{2 i}(M) \cap A^{2 i}$, for $i \leqslant n$, is an orthogonal complement to $Q\left(A^{2 i-4}\right)$, where $Q(\eta)=\eta \wedge\left(\omega_{I}^{2}+\omega_{J}^{2}+\omega_{K}^{2}\right)$. Moreover, $A_{+}^{2 i}$ is irreducible as a representation of $S U(2)$. Therefore, the space $A_{+}^{p, p}=\left.\operatorname{ker} Q^{*}\right|_{A^{p, p}}$ is 1-dimensional.

Proposition 3.2: Let $\left(M, I, J, K, \Phi_{I}\right)$ be an $S L(n, \mathbb{H})$-manifold, equipped with an HKT-structure $\Omega$. Assume that $\Omega^{n}=\Phi_{I}$. Let

$$
\Pi_{+}: \Lambda_{I}^{n+q, n+q}(M) \longrightarrow \Lambda_{I,+}^{n+q, n+q}(M)
$$

be the projection to the component of maximal weight with respect to the $S U(2)$ action. Then $\Xi_{q}:=\Pi_{+}\left(\omega_{I}^{n+q, n+q}\right)$ is a closed, weakly positive $(n+q, n+q)$-form.

Proof: Consider the algebra $A^{*}=\oplus A^{2 i}$ generated by $\omega_{I}, \omega_{J}$, and $\omega_{K}$ as above. The map $R^{p, q}$ is induced by the $S U(2)$-action, hence it maps $A^{*, *}$ to itself. Since $\mathcal{V}_{p, q}(\eta)=\mathcal{R}_{p, q}(\eta) \wedge \mathcal{V}_{0,0}(1)$, and $\mathcal{V}_{0,0}(1)$ is proportional to $\mathcal{R}_{n, n}\left(\Phi_{I}\right) \in A^{*}$, we obtain

$$
\mathcal{V}_{p, q}\left(A^{p+q, 0}\right) \subset A^{n+p, n+q} .
$$

Since $\mathcal{V}\left(\Omega^{p}\right) \subset A_{+}^{n+p, n+p}$, the 1-dimensional space $A_{+}^{n+p, n+p}$ is generated by $\mathcal{V}\left(\Omega^{p}\right)$. This form is closed by Proposition 3.1. Therefore, the projection of $\omega_{I}^{n+p}$ to $A_{+}^{n+p, n+p}$ is closed.

\section{Balanced HKT manifolds}

4.1. Quaternionic Monge-Ampère equation. Let $(M, I, J, K)$ be a hypercomplex manifold, and $\varphi \longrightarrow \partial \partial_{J} \varphi$ the operator $C^{\infty}(M) \stackrel{\partial \partial_{J}}{\longrightarrow} \Lambda_{I}^{2,0}(M)$ defined in Subsection 2.3. In [AV2], the quaternionic Monge-Ampère operator $C^{\infty}(M) \longrightarrow \Lambda_{I}^{2 n, 0}(M)$ was defined, mapping a function $\varphi$ to $\left(\partial \partial_{J} \varphi\right)^{n}$, where $n=\operatorname{dim}_{\mathbb{H}} M$ (see also [A]). This operator is remarkably similar to the usual Monge-Ampère operators (real and complex) which are well known in geometry. A quaternionic version of Calabi-Yau theorem was conjectured.

Conjecture 4.1: Let $(M, I, J, K, \Omega)$ be a compact HKT manifold with holonomy $S L(b, \mathbb{H}), \operatorname{dim}_{\mathbb{H}} M=n$, and $\Phi \in \Lambda_{I}^{2 n, 0}(M)$ a nowhere degenerate real ${ }^{1}$, section of the canonical bundle. Then

$$
\Phi=A\left(\Omega+\partial \partial_{J} \varphi\right)^{n}
$$

\footnotetext{
${ }^{1}$ In the sense of Subsection 2.3
} 
for some constant $A>0$ and a real function $\varphi \in C^{\infty}(M)$.

It is easy to see that in this case, $\Omega+\partial \partial_{J} \varphi$ is an HKT-form (see the proof of Proposition 4.2), hence Conjecture 4.1 implies existence of an HKT-metric $\Omega^{\prime}=$ $\Omega+\partial \partial_{J} \varphi$ such that the corresponding volume form is proportional to $\Phi$.

When $\operatorname{Hol}(M) \subset S L(n, \mathbb{H})$, this conjecture was partly verified in [AV2]. We have shown that the solution of (4.1) is unique, and also gave a priori $C_{0}$-bounds on its solution. For Yau's proof of existence of solutions of Monge-Ampère equation to work, one also needs $C_{2}$ and $C_{3}$-bounds.

Conjecture 4.1 immediately implies the following statement.

Proposition 4.2: Let $(M, I, J, K, \Omega)$ be a compact HKT-manifold with holonomy $\operatorname{Hol}(M) \subset S L(n, \mathbb{H})$, and $\Phi \in \Lambda_{I}^{2 n, 0}(M)$ a non-zero section of the canonical class which is parallel with respect to the Obata connection $\nabla$. Assume that the Conjecture 4.1 is true for $M$. Then $M$ admits an HKT-form $\Omega_{1}=\Omega+\partial \partial_{J} \varphi$ such that $\Omega_{1}^{n}$ is a holomorphic volume form. Moreover, in this case, one has $\nabla\left(\Omega_{1}^{n}\right)=0$, where $\nabla$ is the Obata connection.

Proof: Let $\varphi$ be a solution of an equation $\Phi=A\left(\Omega+\partial \partial_{J} \varphi\right)^{n}$. The form $\Omega_{1}:=$ $\Omega+\partial \partial_{J} \varphi$ is $\mathbb{H}$-positive. Indeed, since $\Phi=A\left(\Omega+\partial \partial_{J} \varphi\right)^{n}$, this form is nowhere degenerate. At a point $p \in M$ where $\varphi$ reaches its minimum, the quaternionic Hessian form $\partial \partial_{J} \varphi$ is positive (Subsection 2.3), hence at $p$ the quaternionic Hermitian form $x, y \longrightarrow \Omega_{1}(x, J y)$ is positive definite. Since $\Omega_{1}$ is nowhere degenerate, this form is positive definite everywhere on $M$. Therefore, $\Omega_{1}$ is an HKT-form. To check that $\nabla\left(\Omega_{1}^{n}\right)=0$, one uses Lemma 4.3 below. We proved Proposition 4.2.

The following lemma is essentially contained in [BDV] (Theorem 3.2).

Lemma 4.3: Let $(M, I, J, K)$ be a hypercomplex manifold, and $\eta$ a top degree $(2 n, 0)$ form, which is $\mathbb{H}$-real and holomorphic. Then $\eta$ is Obata-parallel.

Proof: Since the Obata connection is torsion-free, $d \eta=\operatorname{Alt}(\nabla \eta)$, where Alt $=\Lambda$ : $\Lambda^{2 n}(M) \otimes \Lambda^{1}(M) \longrightarrow \Lambda^{2 n+1}(M)$ denotes the exterior product. Since $\eta$ is holomorphic, $\bar{\partial} \eta=0$. The map Alt restricted to $\Lambda^{2 n, 0}(M) \otimes \Lambda^{0,1}(M)$ is an isomorphism; therefore, $\nabla^{0,1} \eta=0$. Since $\eta$ is real, $J(\bar{\eta})=\eta$, and we have

$$
\nabla^{0,1} J(\bar{\eta})=\nabla^{0,1} \eta=0
$$

This gives $\nabla^{0,1} \bar{\eta}=0$, because $J$ is Obata-invariant. However, $\nabla^{0,1} \bar{\eta}=\overline{\nabla^{1,0} \eta}$, and this gives $\nabla^{1,0} \eta=0$. We proved that $\nabla^{0,1} \eta+\nabla^{1,0} \eta=\nabla \eta=0$.

Remark 4.4: It is quite hard to construct examples of compact HKT-manifolds with holonomy in $S L(n, \mathbb{H})$. So far, the only one construction is known. In [BDV], it was shown that all hypercomplex nilmanifolds have holonomy in $S L(n, \mathbb{H})$. However, for an HKT nilmanifold, one always has a left-invariant HKT-form ([FG]), and such a form satisfies $\nabla\left(\Omega^{n}\right)=0$ ([BDV], Theorem 3.2). Therefore, Proposition 4.2 is true in this situation, though Conjecture 4.1 is not proven even in the simplest cases. 
Remark 4.5: An HKT-form $\Omega_{1}$ satisfying $\Omega_{1}^{n}=\varphi$ is unique in its cohomology class $\left[\Omega_{1}\right] \in H^{2}\left(\mathcal{O}_{(M, I)}\right)$, where $H^{2}\left(\mathcal{O}_{(M, I)}\right)$ denotes the holomorphic cohomology of $(M, I)$. Indeed, as shown in [V3] (see also [AV1]), on any $S L(n, \mathbb{H})$-manifold two closed, real $(2,0)$-forms $\Omega$ and $\Omega^{\prime}$ which belong to the same cohomology class in $H^{2}\left(\mathcal{O}_{(M, I)}\right)$ satisfy $\Omega-\Omega^{\prime}=\partial \partial_{J} \varphi$. However, the equation (4.1) cannot have two different solutuions, as shown in [AV2], Theorem 4.7.

4.2. Balanced HKT-manifolds. Definition 4.6: Let $(M, I, g)$ be a complex Hermitian manifold, $\operatorname{dim}_{\mathbb{C}} M=n$, and $\omega \in \Lambda^{1,1}(M)$ its Hermitian form. One says that $M$ is balanced if $d\left(\omega^{n-1}\right)=0$.

Remark 4.7: It is easy to see that $d\left(\omega^{m}\right)=0$ for $1 \leqslant m \leqslant n-2$ implies that $\omega$ is Kähler; the balancedness makes sense as the only non-trivial condition of form $d\left(\omega^{m}\right)=0$ which is not equivalent to the Kähler property.

Let $(M, I, J, K, \Omega)$ be an HKT-manifold, $\operatorname{dim}_{\mathbb{H}} M=n$. The "HKT Calabi-Yau" condition $\bar{\partial}\left(\Omega^{n}\right)=0$ has an elegant differential-geometric interpretation.

Theorem 4.8: Let $(M, I, J, K, \Omega)$ be an HKT-manifold, $\operatorname{dim}_{\mathbb{H}} M=n$. Then the following conditions are equivalent.

(i): $\bar{\partial}\left(\Omega^{n}\right)=0$

(ii): $\nabla\left(\Omega^{n}\right)=0$, where $\nabla$ is the Obata connection

(iii): The manifold $(M, I)$ with the induced quaternionic Hermitian metric is balanced as a Hermitian manifold:

$$
d\left(\omega_{I}^{2 n-1}\right)=0 .
$$

Remark 4.9: A balanced HKT-manifold has holonomy in $S L(n, \mathbb{H})$. This statement follows immediately from the implication (iii) $\Rightarrow$ (ii) of Theorem 4.8 .

Remark 4.10: The condition $\nabla\left(\Omega^{n}\right)=0$ is independent from the choice of a basis $I, J, K, I J=-J I=K$ of $\mathbb{H}$. Indeed, suppose that $g \in S U(n)$, and $\left(I_{1}, J_{1}, K_{1}\right)=$ $(g(I), g(J), g(K))$ is a new basis in $\mathbb{H}$. The corresponding HKT-form $\Omega_{1}=\omega_{J_{1}}+$ $\sqrt{-1} \omega_{K_{1}}$ can be expressed as $\Omega_{1}=g(\Omega)$, hence

$$
\nabla\left(\Omega_{1}^{n}\right)=\nabla\left(g\left(\Omega_{1}^{n}\right)\right)=g\left(\nabla\left(\Omega^{n}\right)\right)=0 .
$$

Therefore, Theorem 4.8 leads to the following corollary.

Corollary 4.11: Let $(M, I, J, K, \Omega)$ be an HKT-manifold, such that the corresponding complex Hermitian manifold $(M, I)$ is balanced. Then $\left(M, I_{1}\right)$ is balanced for any complex structrure $I_{1}$ induced by the quaternions. Moreover, $(M, I, J, K, \Omega)$ is an $S L(\mathbb{H}, n)$-manifold.

Proof of Theorem 4.8: The equivalence (i) $\Leftrightarrow$ (ii) is immediate from Lemma 4.3. The implication (ii) $\Rightarrow$ (iii) easily follows from Proposition 3.1. Indeed, $\Omega^{n-1}$ is $\partial$ - and $\partial_{J}$-closed, hence $\mathcal{V}\left(\Omega^{n-1}\right)$ is a closed $(2 n-1,2 n-1)$-form. This form is proportional to $\omega_{I}^{2 n-1}$, by Proposition 3.2.

The implication (iii) $\Rightarrow$ (i) is proven as follows. 
Step 1: The Hermitian manifold $\left(M, I, \omega_{I}\right)$ is balanced if and only if $d^{*} \omega_{I}=0$, which is equivalent to $\partial^{*} \omega_{I}=\bar{\partial}^{*} \omega_{I}=0$. By Theorem 2.5, this is equivalent to $\partial^{*} \Omega=$ $\partial_{J}^{*} \Omega=0$. This gives $d^{*} \Omega=0$. We obtain that $d^{*} \omega_{I}=0$ leads to $d^{*} \omega_{J}=d^{*} \omega_{K}=0$. This also brings

$$
d\left(\Omega^{n} \wedge \bar{\Omega}^{n-1}\right)=* d^{*} \Omega=0 .
$$

Step 2: Let $\bar{\theta}$ be a $(0,1)$-form defined by

$$
\bar{\partial}\left(\Omega^{n}\right)=\Omega^{n} \wedge \bar{\theta} .
$$

Using a (2,0)-version of Lefschetz $\mathfrak{s l}(2)$-action ([V3]), it is easy to observe that the map

$$
\Lambda_{I}^{0,1}(M) \stackrel{\wedge \bar{\Omega}^{n-1}}{\longrightarrow} \Lambda_{I}^{0, n-1}(M)
$$

is an isomorphism. The HKT-condition gives $\overline{\partial \Omega}=0$, hence

$$
0=\bar{\partial}\left(\Omega^{n} \wedge \bar{\Omega}^{n-1}\right)=\bar{\partial}\left(\Omega^{n}\right) \wedge \bar{\Omega}^{n-1}=\Omega^{n} \wedge \bar{\theta} \wedge \bar{\Omega}^{n-1} .
$$

Using the isomorphism (4.2), we obtain that $\Omega^{n} \wedge \bar{\theta} \wedge \bar{\Omega}^{n-1}=0$ implies that $\bar{\theta}=0$. Therefore, $\bar{\partial}\left(\Omega^{n}\right)=0$. The implication (iii) $\Rightarrow$ (i) is proven. We have finished the proof of Theorem 4.8

Remark 4.12: From Remark 4.5, we obtain that a balanced HKT-metric on an HKT-manifold is unique in its cohomology class $[\Omega] \in H^{2}\left(\mathcal{O}_{(M, I)}\right)$. In particular, the space of balanced HKT-metrics is finite-dimensional.

Proposition 4.13: Let $(M, I, J, K, \Omega)$ be a balanced HKT manifold, and $\Xi_{k}:=$ $\Pi_{+}\left(\omega_{I}^{k}\right)$ the maximal weight component of $\omega_{I}^{k}$. Then $d \Xi_{k}=0$ for any $k \geqslant n$, where $n=\operatorname{dim}_{\mathbb{H}} M$. Moreover, the $(k, k)$-form $\Xi_{k}$ is weakly positive.

Proof: On a balanced HKT-manifold $(M, I, J, K, \Omega)$, the top exterior power $\Omega^{n}$ is Obata parallel and holomorphic (Theorem 4.8). Now, Proposition 4.13 is directly implied by Proposition 3.2.

\section{Strong HKT manifolds}

5.1. Strong HKT metrics and HKT-potential. There is another important class of HKT metrics, called strong HKT metrics. For physicists, such metrics are of special interest ([GHR], [HP]). The original definition of HKT metrics $([\mathrm{HP}])$ assumed the strong HKT condition; in mathematical literature it was dropped, because of relative lack of examples.

Definition 5.1: Let $(M, I, J, K)$ be a hypercomplex manifold, and $g$ a quaternionic Hermitian metric on $M$. The metric $g$ is called strong HKT (abbreviated for sHKT) if it is HKT and, moreover,

$$
d d^{c} \omega_{I}=0
$$

where $d^{c}:=-I d I$ is the usual twisted differential. 
Remark 5.2: Let $(M, I, J, K, g)$ be an HKT-manifold. The torsion of the Bismut connection on $M$ can be written as

$$
T=-I d \omega_{I}=-J d \omega_{J}=-K d \omega_{K}
$$

(Remark 1.2). Clearly, the sHKT condition is equivalent to $d T=0$.

Remark 5.3: Let $(M, I, J, K)$ be a hypercomplex manifold equipped with a quaternionic Hermitian structure. Denote by $H$ the $S U(2)$-representation generated by the 3 -forms $d \omega_{I}, d \omega_{J}, d \omega_{K}$. The HKT condition is equivalent to $\operatorname{dim} H \leqslant 4$ (Remark 1.2). On top of it, the strong HKT condition means that for any $v \in H$, one has $d v=0$. Indeed, $g$ is HKT if and only if

$$
I d \omega_{I}=J d \omega_{J}=K d \omega_{K} .
$$

Therefore, $H$ is generated by four 3 -forms

$$
H=\left\langle d \omega_{I}, \quad I d \omega_{I}, \quad J d \omega_{I}, \quad K d \omega_{I}\right\rangle .
$$

Writing $d \omega_{I}=-K d \omega_{J}=J d \omega_{K}$, we obtain $J d \omega_{I}=-d \omega_{K}, K d \omega_{I}=d \omega_{J}$. This allows us to rewrite the basis (5.2), for any HKT-manifold:

$$
H=\left\langle d \omega_{I}, \quad I d \omega_{I}, \quad J d \omega_{I}=-d \omega_{K}, \quad K d \omega_{I}=d \omega_{J}\right\rangle .
$$

Of those 43 -forms, 3 are manifestly exact, and $I d \omega_{I}$ is closed if and only if $M$ is sHKT, as follows from Remark 5.2.

Proposition 5.4: Let $(M, I, J, K)$ be an HKT-manifold, and $\Omega \in \Lambda_{I}^{2,0}(M)$ its HKT form. Then the strong HKT condition is equivalent to $\overline{\partial \partial}_{J} \Omega=0$.

Proof: Clearly, $\bar{\partial}_{J} \Omega$ is an element in $H$, and for all $v \in H$, and all differentials $\delta$ of form $d, I d I, J d J, K d K$, we have $\delta(v)=0$ per Remark 5.3. Therefore, strong HKT implies $\overline{\partial \partial}_{J} \Omega=0$.

To prove the converse implication, consider a local potential $\varphi$ for $\Omega, \Omega=\partial \partial_{J} \varphi$, where $\varphi \in C^{\infty}(M)$ is a smooth function, defined locally on $M$. Such a potential exists as shown in [BS] (see also [AV1]). Then $\overline{\partial \partial}_{J} \Omega=0$ is equivalent to

$$
\overline{\partial \partial}_{J} \partial \partial_{J} \varphi=0 \text {. }
$$

The operators $\partial, \partial_{J}, \bar{\partial}, \bar{\partial}_{J}$ can be written down as linear combinations of twisted differentials $d, d_{I}:=-I d I, d_{J}:=-J d J, d_{K}:=-K d K$. These differentials pairwise anticommute ([V3]), hence the only non-zero homogeneous fourth order monomial expressed through $d, d_{I}, d_{J}, d_{K}$ is the operator $\Lambda^{*}(M) \stackrel{d d_{I} d_{J} d_{K}}{\longrightarrow} \Lambda^{*+4}(M)$. This gives

$$
\overline{\partial \partial}_{J} \partial \partial_{J}=\text { const } \cdot d d_{I} d_{J} d_{K},
$$

where const is a non-zero constant which can be found by an explicit calculation. The Hermitian form $\omega_{I}$ can be expressed through $\varphi$ as

$$
\omega_{I}=d d_{I} \varphi+d_{J} d_{K} \varphi
$$

([BS]). Therefore, the strong HKT condition is equivalent to

$$
0=d d_{I} \omega=d d_{I} d_{J} d_{K} \varphi=0
$$


This is equivalent to $\overline{\partial \partial}_{J} \partial \partial_{J} \varphi=0$ as follows from (5.4). We proved Proposition 5.4.

Remark 5.5: The strong HKT condition can be expressed through the potential, as indicated above. An HKT metric with potential $\varphi$ is strong HKT if and only if $\varphi$ satisfies $d d_{I} d_{J} d_{K} \varphi=0$.

5.2. HKT classes and strong HKT metrics. Let $M$ be a hypercomplex manifold. Consider the complex

$$
C^{\infty}(M) \stackrel{\partial \partial_{J}}{\longrightarrow} \Lambda_{I}^{2,0}(M) \stackrel{\partial \oplus \partial_{J}}{\longrightarrow} \Lambda_{I}^{3,0}(M) \oplus \Lambda_{I}^{3,0}(M) .
$$

It is easy to see that this complex is elliptic ([AV1]).

Denote the cohomology of the complex (5.6) by $H_{\partial \partial_{I}}^{2,0}(M)$. From Theorem 2.5 it follows immediately that the group $H_{\partial \partial_{J}}^{2,0}(M)$ is independent from the choice of a quaternionic triple $I, J, K$.

This group is similar to the Bott-Chern cohomology group in complex geometry ([Te], $[\mathrm{OV}])$. The Bott-Chern cohomology encodes the cohomological information about holomorphic line bundles, and the group $H_{\partial \partial_{J}}^{2,0}(M)$ encodes the cohomological information about the HKT-forms.

Definition 5.6: Let $(M, I, J, K, \Omega)$ be an HKT manifold, and $[\Omega] \in H_{\partial \partial_{J}}^{2,0}(M)$ the cohomology class of the HKT form $\Omega$. Then $[\Omega]$ is called the HKT class of $M$.

Clearly, HKT forms $\Omega, \Omega_{1}$ have the same HKT class if and only if $\Omega-\Omega_{1}=\partial \partial_{J} \varphi$, for a smooth function $\varphi$, globally defined on $M$.

Remark 5.7: Using Hodge theory (in particular, the $\partial \bar{\partial}$-lemma) one can prove that the Bott-Chern cohomology of a compact Kähler manifold $X$ is equal to the usual Hodge cohomology group $H^{1,1}(X)$. For compact HKT-manifolds with holonomy in $S L(n, \mathbb{H})$ a version of Hodge theory was proven in [V3]. In particular, it was shown that for any compact HKT-manifold with $\operatorname{Hol}(M) \subset S L(n, \mathbb{H})$, the natural map $H_{\partial \partial_{J}}^{2,0}(M) \longrightarrow H_{\partial}^{2}\left(\Lambda^{*, 0}(M)\right) \cong \overline{H_{\partial}^{2}\left(\mathcal{O}_{(M, I)}\right)}$ to the complex conjugate to the corresponding holomorphic cohomology is an isomorphism.

Remark 5.8: Let $(M, I, J, K)$ be a hypercomplex manifold, $\operatorname{dim}_{\mathbb{H}} M=n$, and $g$ a quaternionic Hermitian form (such a form always exists). Denote by $\omega_{I}, \omega_{J}$ and $\omega_{K}$ the corresponding Hermitian forms (1.1). Consider the 4-th order operator from $C^{\infty}(M)$ to $C^{\infty}(M)$,

$$
\varphi \stackrel{\square}{\longrightarrow}\left(d d_{I} d_{J} d_{K} \varphi, \omega_{I}^{2}+\omega_{J}^{2}+\omega_{K}^{2}\right),
$$

where $(\cdot, \cdot)$ denotes the Hermitian product on differential forms induced from $g$. Using the usual Kähler identities, one immediately obtains that on a hyperkähler manifold, $\square=\Delta^{2}$, where $\Delta$ is the Laplacian. Therefore, $\square$ is elliptic, and the equation $d d_{I} d_{J} d_{K} \varphi=0, \varphi \in C^{\infty}(M)$ is overdetermined.

This leads to the following conjecture.

Conjecture 5.9: Let $(M, I, J, K)$ be a compact HKT manifold, and $[\Omega] \in H_{\partial \partial_{J}}^{2,0}(M)$ its HKT class. Then there exists at most one strong HKT form $\Omega^{\prime}$ with $\left[\Omega^{\prime}\right]=[\Omega]$. 
Remark 5.10: For $M$ hyperkähler, Conjecture 5.9 is clear. Indeed, if strong HKT forms $\Omega, \Omega^{\prime}$ have the same cohomology class, one has $\Omega-\Omega^{\prime}=\partial \partial_{J} \varphi$, and (as shown in the proof of Proposition 5.4), this brings $d d_{I} d_{J} d_{K} \varphi=0$. Using Remark 5.8, one obtains that $\Delta^{2} \varphi=0$, hence $\varphi$ is constant. In particular, any strong HKT metric on a compact hyperkähler manifold is automatically hyperkähler.

5.3. Strong HKT metrics on balanced HKT manifolds. A complex Hermitian manifold $(M, I, \omega)$ is called strong $\mathbf{K T}$ if it satisfies $d d^{c} \omega=0$. It is balanced if $d^{*} \omega=0$. In [AI], Section 2, Remark 1, and independently in [FPS], Proposition 1.4, it was shown that a compact Hermitian manifold which is balanced can never be strong KT, unless it is Kähler. A stronger result can be proven for balanced HKTmanifolds, following the same lines, if one uses the $S p(1,1)$-representation theory as indicated below.

Theorem 5.11: Let $(M, I, J, K, \Omega)$ be a balanced HKT-manifold. Assume that $n=$ $\operatorname{dim}_{\mathbb{H}} M \geqslant 3$. If $M$ admits a strong HKT-form in the same cohomology class $[\Omega] \in$ $H^{2}\left(\mathcal{O}_{(M, I)}\right)$, then $d \Omega=0$, and $M$ is hyperkähler.

Proof: The balanced HKT condition is equivalent to $\Lambda_{\omega_{I}} d \omega_{I}=0$, where $\Lambda_{\omega_{I}}$ is the Hermitian adjoint to $\eta \longrightarrow \eta \wedge \omega_{I}$. Indeed,

$$
\Lambda_{\omega_{I}} d \omega_{I}=\frac{* d\left(\omega_{I}^{2 n-1}\right)}{2 n-1}
$$

Forms satisfying $\Lambda_{\omega_{I}} \eta=0$ are called primitive.

Denote by $\Xi_{3} \in \Lambda_{I}^{2 n-3,2 n-3}(M)$ the maximum weight component of $\omega_{I}^{2 n-3}$. By Proposition $4.13, \Xi_{3}$ is a closed, positive $(2 n-3,2 n-3)$-form.

The $(2,1)$-Hodge component $\left(d \omega_{I}\right)^{2,1} \in \Lambda_{I}^{2,1}(M)$ is closed by Remark 5.3 , primitive as seen above, and has weight 1 with respect to $S U(2)$ as follows from Theorem 2.5. Therefore, $\left(d \omega_{I}\right)^{2,1}$ is a highest weight vector with respect to $\mathfrak{s o}(1,4)$-action associated with the quaternionic Hermitian structure ([V0]; see Subsection 6.1 for details). By Theorem 6.2, the Hermitian form

$$
\eta_{1}, \eta_{2} \longrightarrow \sqrt{-1} \int_{M} \eta_{1} \wedge \bar{\eta}_{2} \wedge \Xi_{3}
$$

is sign-definite on the space of primitive $(2,1)$-forms of weight 1 . Therefore,

$$
\int_{M}\left(d \omega_{I}\right)^{2,1} \wedge\left(d \omega_{I}\right)^{1,2} \wedge \Xi_{3} \neq 0
$$

unless $\left(d \omega_{I}\right)^{2,1}=0$. A trivial calculation gives

$$
\left(d \omega_{I}\right)^{2,1} \wedge\left(d \omega_{I}\right)^{1,2}=\sqrt{-1} d \omega_{I} \wedge d_{I} \omega_{I} .
$$

Since

$$
0=\int_{M} d d_{I}\left(\omega_{I} \wedge \omega_{I} \wedge \Xi_{3}\right)=\int_{M} d \omega_{I} \wedge d_{I} \omega_{I} \wedge \Xi_{3}+\int_{M} d d_{I} \omega_{I} \wedge \omega_{I} \wedge \Xi_{3}
$$

we conclude that

$$
\int_{M} d d_{I} \omega_{I} \wedge \omega_{I} \wedge \Xi_{3} \neq 0
$$


unless $(M, I, J, K, \Omega$ is hyperkähler.

Now assume that $(M, I, J, K)$ admits an sHKT-form $\Omega^{\prime}$ in the same cohomology class. Then $\Omega-\Omega^{\prime}=\partial \partial_{J} \varphi$ for some globally defined function $\varphi$ on $M$. Since $\overline{\partial \partial}_{J} \Omega^{\prime}=0$,

$$
\overline{\partial \partial}_{J} \partial \partial_{J} \varphi=\overline{\partial \partial}_{J}\left(\Omega-\Omega^{\prime}\right)=\overline{\partial \partial}_{J} \Omega .
$$

From (5.4), we obtain that

$$
d d_{I} d_{J} d_{K} \varphi=\text { const } \overline{\partial \partial}_{J} \partial \partial_{J} \varphi=\text { const } \overline{\partial \partial}_{J} \Omega=d d_{I} \omega_{I},
$$

where const is a non-zero rational number. Then (5.7) gives

$$
0 \neq \int_{M} d d_{I} \omega_{I} \wedge \omega_{I} \wedge \Xi_{3}=\int_{M} d d_{I} d_{J} d_{K} \varphi \wedge \omega_{I} \wedge \Xi_{3} .
$$

Since $\Xi_{3}$ is $d, d_{I}, d_{J}, d_{K}$-closed, the last integral can be rewritten as

$$
\int_{M} d d_{I} d_{J} d_{K} \varphi \wedge \omega_{I} \wedge \Xi_{3}=\int_{M} \varphi d d_{I} d_{J} d_{K} \omega_{I} \wedge \Xi_{3} .
$$

However, locally $\omega_{I}$ is written as and $\omega_{I}=d d_{I} \psi+d_{J} d_{K} \psi$ by Banos-Swann (5.5), hence the form $d d_{I} d_{J} d_{K} \omega_{I}$ is identically zero. Therefore, the integral (5.7) must vanish as well, and $(M, I, J, K, \Omega)$ must be hyperkähler.

Remark 5.12: If Conjecture 4.1 is true, then every $S L(n, \mathbb{H})$-manifold contains a balanced HKT-metric in each HKT-class, necessarily unique. Then Theorem 5.11 implies that an $S L(n, \mathbb{H})$-manifold admits no sHKT-metrics, for $n \geqslant 3$.

5.4. Strong HKT metrics on nilmanifolds. The only examples (so far) of compact $S L(n, \mathbb{H})$-manifolds are hypercomplex nilmanifolds. A hypercomplex nilmanifold is a quotient of a nilpotent Lie group equipped with a left-invariant hypercomplex structure, by a co-compact, discrete subgroup, acting from the left. In [FG] it was shown that any HKT-metric on a hypercomplex nilmanifold can be averaged with the Lie group action, giving rise to a left-invariant HKT-metric. The left-invariant HKT-structures can be considered as metrics on the corresponding Lie algebra, and studied algebraically.

The HKT-metrics on hypercomplex nilmanifolds are studied in [BDV]. It was shown that a hypercomplex nilmanifold which admits an HKT metric is necessarily abelian, that is, the corresponding Lie subalgebra $\mathfrak{g}^{1,0}$ of left-invariant $(1,0)$-vector fields is abelian. In [DF], Proposition 2.1 it was shown that any abelian hypercomplex nilmanifold admitting a strong HKT metric is necessarily a torus. Therefore, for nilmanifolds Theorem 5.11 is known from conjunction of [BDV] and [DF].

The problem of constructing strong HKT-manifolds seems to be difficult. All compact non-hyperkähler examples of sHKT-manifolds known so far are homogeneous. The hypercomplex structures on semisimple Lie groups obtained by physicists $\mathrm{Ph}$. Spindel et al ([SSTV]) and independently by D. Joyce ([J]) are strong HKT ([GP]). A powerful new method of "doubling" a strong HKT $4 n$-dimensional Lie algebra to obtain a strong HKT Lie algebra of dimension $8 n$ is proposed in $[\mathrm{BF}]$. 


\section{Appendix: Hyperkähler Hodge-Riemann relations}

6.1. $\mathfrak{s o}(4,1)$-action and the Schur's lemma. Let $V$ be a quaternionic Hermitian vector space, and $\Lambda^{*} V$ its exterior algebra. Consider the 2 -forms $\omega_{I}, \omega_{J}, \omega_{K}$, defined on $V$ as in (1.1). Let $\mathfrak{a}$ be the Lie algebra generated by the operators

$$
L_{\omega_{I}}(\eta)=\eta \wedge \omega_{I}, \quad L_{\omega_{J}}(\eta)=\eta \wedge \omega_{J}, \quad L_{\omega_{K}}(\eta)=\eta \wedge \omega_{K},
$$

and their Hermitian adjoints. In [V0], it was shown that $\mathfrak{a} \cong \mathfrak{s o}(4,1)=\mathfrak{s} \mathfrak{p}(1,1)$ (in [V2], the corresponding Lie group was found; it is isomorphic to $S p(1,1) \cong \operatorname{Spin}(4,1))$.

The Lie algebra $\mathfrak{s p}(1,1)$ has rank 2 . As shown in [V0], one could choose a Cartan subalgebra of $\mathfrak{a}$ in such a way that the corresponding weight decomposition of $\Lambda^{*} V$ coincides with the Hodge decomposition $\Lambda^{*} V=\bigoplus_{p, q} \Lambda^{p, q}(V)$.

Definition 6.1: Let $A$ be a vector space, and $\mathfrak{g}$ a Lie algebra acting on $A$. Assume that $A$ is a semisimple representation, that is, $A$ is a direct sum of irreducible $\mathfrak{g}$-representations. Consider a decomposition $A=\bigoplus A_{\alpha}$ of $A$ into a direct sum of representations of $\mathfrak{g}$, with each $A_{\alpha}$ being a sum of isomorphic irreducible representations, non-isomorphic between different $A_{\alpha}$. Such a decomposition is called the isotypic decomposition; it is obviously unique and well-defined.

Let $\Lambda^{*} V=\oplus_{\alpha} I_{\alpha}$ be the isotypic decomposition of $\Lambda^{*} V$ with respect to the action of $\mathfrak{a}$. Since the Hodge decomposition on $\Lambda^{*} V$ is induced by the $\mathfrak{s p}(1,1)$-action, the Hodge decomposition of $\Lambda^{*} V$ is compatible with the isotypic decomposition. Let $I_{\alpha}=\oplus_{p, q} I_{\alpha}^{p, q}$ be the Hodge decomposition of $I_{\alpha}$, taken with respect to the complex structure $I$ on $V$.

The main result of this Appendix is the following quaternionic Hermitian version of Hodge-Riemann relations.

Theorem 6.2: Let $V=\mathbb{H}^{n}$ be a quaternionic Hermitian space, and $\Lambda^{*} V=\oplus I_{\alpha}^{p, q}$ the Hodge decomposition of its isotypic decomposition defined above. Consider a form $P \in \Lambda^{2 n-k, 2 n-k} V, P=P\left(\omega_{I}, \omega_{J}, \omega_{K}\right)$ obtained as an order $(2 n-k)$ homogeneous polynomial of $\omega_{I}, \omega_{J}, \omega_{K}$, and let $(\cdot, \cdot)_{P}$ be a semi-linear pairing on $I_{\alpha}^{p, q}$, defined as

$$
\left(\eta, \eta^{\prime}\right)_{P}:=\frac{\eta \wedge \bar{\eta}^{\prime} \wedge P}{\operatorname{Vol} V}
$$

where $p+q=k$ and $\mathrm{Vol} V$ is the Riemannian volume form on $V$. Then $(\cdot, \cdot)_{P}$ is sign-definite or identically zero.

Theorem 6.2 is an immediate consequence of Schur's lemma together with the following theorem, proven in Subsection 6.2.

Theorem 6.3: Let $V=\mathbb{H}^{n}$ be a quaternionic Hermitian space, and $\Lambda^{*} V=\oplus I_{\alpha}^{p, q}$ the Hodge decomposition of its isotypic decomposition defined above. Denote by $S p(V)$ the group $S p(n)$ of quaternionic unitary matrices acting on $V$; this group obviously commutes with $S p(1,1)$-action, hence preserves the spaces $I_{\alpha}^{p, q}$. Then the spaces $I_{\alpha}^{p, q}$ are irreducible as representations of $S p(V)$, for all $p, q, \alpha$. 
Remark 6.4: Let $W=\mathbb{C}^{n}$ be a Hermitian vector space, $\Lambda^{*} V$ its (real) exterior algebra, equipped with a usual Lefschetz-type $\mathfrak{s l}(2)$-action, $\Lambda^{*} V=\oplus I_{\alpha}$ its isotypic decomposition, and $\Lambda^{*} V=\oplus I_{\alpha}^{p, q}$ the Hodge decomposition of $\oplus I_{\alpha}$. The HodgeRiemann relations state that the form

$$
\left(\eta, \eta^{\prime}\right):=\frac{\eta \wedge \bar{\eta}^{\prime} \wedge \omega^{k}}{\operatorname{Vol} V}
$$

is sign-definite on the space $I_{\alpha}^{p, q}$, where $p+q+2 k=n$. It is deduced directly from Schur's lemma, because, as follows from Howe's duality, the spaces $I_{\alpha}^{p, q}$ are irreducible as representations of $U(n)([\mathrm{Ho}])$, and the form $(\cdot, \cdot)$ is $U(n)$-invariant.

The hyperkähler Hodge-Riemann relations are proven using the same argument, with $S p(n)$ instead of $U(n)$.

Remark 6.5: On a compact hyperkähler manifold $M$, the $S p(1,1)$-action preserves the harmonic forms. Therefore, the decomposition $\Lambda^{*} M=\oplus I_{\alpha}^{p, q}$ is well defined on harmonic forms. The forms $P=P\left(\omega_{I}, \omega_{J}, \omega_{K}\right)$ which can be expressed polynomially through $\omega_{I}, \omega_{J}, \omega_{K}$ are closed, hence the pairing $(\cdot, \cdot)$ is well defined in cohomology. In this situation, the Hodge-Riemann relations have topological interpretation, similar to the Hodge index theorem in the Kähler case.

6.2. Howe's duality and $\mathfrak{s p}(1,1)$-action. Howe's duality can be stated as in R. Howe's paper [Ho] in a very general fashion involving graded Clifford algebras associated with graded vector spaces. This version of Howe's duality includes both the usual Clifford algebra and usual spinors and its odd counterpart, the Weil algebra (the algebra of differential operators) and the Weil representation, also known as the space of symplectic spinors.

To obtain the hyperkähler Hodge-Riemann relations, the symplectic spinorial part of this picture is not needed.

To simplify the exposition, we omit the odd Clifford part of the statement, and state the Howe's duality for usual Clifford algebras and the usual spinors.

Let $V$ be a vector space, $W=V \oplus V \oplus V \oplus \ldots$ a sum of several copies of $V$, and $\Lambda^{*} W$ the corresponding Grassmann algebra. Denote by $\tilde{W}$ the sum $W \oplus W^{*}$ equipped with a natural symmetric pairing. The corresponding Clifford algebra $\mathrm{Cl}(\tilde{W})$ is naturaly identified with $\operatorname{End}\left(\Lambda^{*}(W)\right)$, and $\Lambda^{*}(W)$ is identified with the associated spinor space of $\tilde{W}$.

Definition 6.6: In these assumptions, let $\Gamma, \Gamma^{\prime} \subset \mathfrak{o}(\tilde{W})$ be Lie subalgebras of the orthogonal Lie algebra $\mathfrak{o}(\tilde{W})$. The pair $\left(\Gamma, \Gamma^{\prime}\right)$ is called a dual pair (a Howe's dual pair) if $\Gamma$ is a centralizer of $\Gamma^{\prime}$ and $\Gamma^{\prime}$ is a centralizer of $\Gamma$. If $\Gamma$ and $\Gamma^{\prime}$ are reductive Lie algebras, $\left(\Gamma, \Gamma^{\prime}\right)$ is called a reductive dual pair.

The most important example of a dual pair is provided by the following general construction.

Proposition 6.7: ([Ho]) Let $G$ be a classical Lie group, $V$ its fundamental representation, $W=V \oplus V \oplus V \oplus \ldots$, and $\tilde{W}=W \oplus W^{*}$. Denote by $\Gamma \subset \mathfrak{o}(\tilde{W})$ the Lie algebra 
of $G$ acting on $\tilde{W}$, and let $\Gamma^{\prime} \subset \mathfrak{o}(\tilde{W})$ be its centralizer. Then $\left(\Gamma, \Gamma^{\prime}\right)$ is a reductive dual pair.

Such a dual pair is called a classical dual pair.

The Lie algebra $\mathfrak{o}(\tilde{W})$ acts on the corresponding spinor space, hence we can consider $\mathfrak{o}(\tilde{W})$ as a Lie subalgebra in $\mathrm{Cl}(\tilde{W})$.

The main result of Howe's duality is the following useful theorem.

Theorem 6.8: (Howe's duality) Let $G$ be a classical Lie group, $V$ its fundamental representation, and $W=V \oplus V \oplus V \oplus \ldots$. Consider the corresponding classical dual pair $\left(\Gamma, \Gamma^{\prime}\right) \subset \mathfrak{o}(\tilde{W}) \subset \mathrm{Cl}(\tilde{W})$. Then the associative subalgebra of $\mathrm{Cl}(\tilde{W})=\operatorname{End}\left(\Lambda^{*}(W)\right)$ generated by $\Gamma^{\prime}$ is the full algebra of invariants of $G$ in $\mathrm{Cl}(\tilde{W})$.

Proof: This is [Ho], Theorem 7.

To prove Theorem 6.3, let's apply Howe's duality to $G=S p(V), W=V$. The centralizer of $G$ in $\mathfrak{o}(\tilde{W})$ is naturally identified with $\mathfrak{u}(1,1, \mathbb{H})=\mathfrak{s p}(1,1)$, hence $\mathfrak{s p}(1,1)$ generates the full algebra of invariants of $S p(V)$ acting on $\Lambda^{*}(V)$. Since the Cartan algebra action of $\mathfrak{s p}(1,1)$ coincides with the Hodge decomposition of $\Lambda^{*}(V)$, the corresponding eigenspaces $I_{\alpha}^{p, q}$ have no endoporphisms generated by $\mathfrak{s p}(1,1)$. By Howe's duality, this implies that all $I_{\alpha}^{p, q}$ are irreducible representations of $S p(V)$. We proved Theorem 6.3.

Acknowledgements: I am grateful to Ruxandra Moraru and Semyon Alesker for their interest and valuable discussions, and to Victor Ginzburg, Dmitri Kaledin and David Kazhdan for an advice on representation theory. Special thanks to Isabel Dotti, Geo Grantcharov and Stefan Ivanov and for insightful comments and reference.

\section{References}

[AI] Bogdan Alexandrov, Stefan Ivanov, Vanishing theorems on Hermitian manifolds, arXiv:math/9901090, Diff. Geom. Appl. vol. 14 (2001), 251-263.

[A] Semyon Alesker Quaternionic Monge-Ampère equations, arXiv:math/0208005, J. Geom. Anal. 13 (2003), no. 2, 205-238.

[AV1] Semyon Alesker, Misha Verbitsky, Plurisubharmonic functions on hypercomplex manifolds and HKT-geometry, arXiv:math/0510140, J. Geom. Anal. 16 (2006), no. 3, 375-399.

[AV2] Semyon Alesker, Misha Verbitsky Quaternionic Monge-Ampère equation and Calabi problem for HKT-manifolds, arXiv:0802.4202, 32 pages.

[BS] Banos, Bertrand; Swann, Andrew; Potentials for hyper-Kähler metrics with torsion, arXiv:math/0402366, Classical Quantum Gravity 21 (2004), no. 13, 3127-3135.

[BDV] Maria L. Barberis, Isabel G. Dotti, Misha Verbitsky, Canonical bundles of complex nilmanifolds, with applications to hypercomplex geometry, arXiv:0712.3863, 22 pages.

[BF] M. L. Barberis, A. Fino New strong HKT manifolds arising from quaternionic representations, arXiv:0805.2335, 18 pages.

[Bes] Besse, A., Einstein Manifolds, Springer-Verlag, New York (1987)

[CS] Capria, M. M., Salamon, S. M. Yang-Mills fields on quaternionic spaces, Nonlinearity 1 (1988), no. 4, 517-530.

[DF] I. Dotti, A. Fino, Hyperkähler torsion structures invariant by nilpotent Lie groups, Class. Quantum Gravity 19 (2002), 551-562, math.DG/0112166.

[FG] Fino, A., Grantcharov, G., On some properties of the manifolds with skew-symmetric torsion and holonomy $S U(n)$ and $S p(n)$, math.DG/0302358, Adv. Math. 189 (2004), no. 2, 439-450. 
[FPS] Anna Fino, Maurizio Parton, Simon Salamon Families of strong KT structures in six dimensions, math/0209259, Commentarii Mathematici Helvetici (2004), 2, 317-340.

[GHR] Gates, S. J., Jr.; Hull, C. M.; Roček, M., Twisted multiplets and new supersymmetric nonlinear $\sigma$-models, Nuclear Phys. B 248 (1984), no. 1, 157-186.

[G] P. Gauduchon, Hermitian connections and Dirac operators, Bollettino U. M. I. B 11 (1997) 257-288.

[GP] Grantcharov, G., Poon, Y. S., Geometry of hyper-Kähler connections with torsion, math.DG/9908015, Comm. Math. Phys. 213 (2000), no. 1, 19-37.

[J] D. Joyce, Compact hypercomplex and quaternionic manifolds, J. Differential Geom. 35 (1992) no. 3, 743-761

[HP] P.S. Howe, G. Papadopoulos, Twistor spaces for hyper-Kähler manifolds with torsion Phys. Lett. B 379 (1996), no. 1-4, 80-86.

[Ho] Howe, Roger, Remarks on classical invariant theory Trans. Amer. Math. Soc. 313 (1989), no. $2,539-570$.

[Hu] Humphreys, J., Introduction to Lie Algebras and Representation Theory, Graduate Texts in Mathematics, Springer-Verlag, no. 9, 1972.

[MS] S. Merkulov, L. Schwachhöfer, Classification of irreducible holonomies of torsion-free affine connections, math.DG/9907206, Ann. of Math. (2) 150 (1999), no. 1, 77-149, also see Addendum: math.DG/9911266, Ann. of Math. (2) 150 (1999), no. 3, 1177-1179

[Ob] Obata, M., Affine connections on manifolds with almost complex, quaternionic or Hermitian structure, Jap. J. Math., 26 (1955), 43-79.

[OV] Liviu Ornea, Misha Verbitsky, Morse-Novikov cohomology of locally conformally Kähler manifolds, arXiv:0712.0107, 22 pages.

[SSTV] Ph. Spindel, A. Sevrin, W. Troost, A. Van Proeyen Extended supersymmetric $\sigma$-models on group manifolds, Nucl. Phys. B308 (1988) 662-698.

[SSTV] A. Strominger, Superstrings with torsion, Nuclear Phys. B 274 (1986), no. 2, 253-284.

[Te] A. Teleman, The pseudo-effective cone of a non-Kählerian surface and applications, Math. Ann. 335 (2006), no. 4, 965-989.

[V0] M. Verbitsky, On the action of the Lie algebra $\mathfrak{s o ( 5 )}$ on the cohomology of a hyperkähler manifold, Func. Anal. and Appl. 24 (1990), 70-71.

[V1] Verbitsky, M., Cohomology of compact hyperkähler manifolds, arXiv:alg-geom/9501001, 87 pages.

[V2] Verbitsky, M., Mirror Symmetry for hyperkähler manifolds, alg-geom/9512195, Mirror symmetry, III (Montreal, PQ, 1995), 115-156, AMS/IP Stud. Adv. Math., 10, Amer. Math. Soc., Providence, RI, 1999

[V3] Verbitsky, M., Hyperkähler manifolds with torsion, supersymmetry and Hodge theory, math.AG/0112215, Asian J. Math. Vol. 6, No. 4, pp. 679-712 (2002).

[V4] Verbitsky, M., Hyperholomorpic connections on coherent sheaves and stability, 40 pages, math.AG/0107182

[V5] Verbitsky, M., Hyperkähler manifolds with torsion obtained from hyperholomorphic bundles, Math. Res. Lett. 10 (2003), no. 4, 501-513, also in math.DG/0303129.

[V6] M. Verbitsky, Hypercomplex manifolds with trivial canonical bundle and their holonomy, arXiv:math/0406537, "Moscow Seminar on Mathematical Physics, II", American Mathematical Society Translations, 2, 221 (2007).

[V7] M. Verbitsky, Quaternionic Dolbeault complex and vanishing theorems on hyperkahler manifolds, math/0604303, Compos. Math. 143 (2007), no. 6, 1576-1592.

[V8] Verbitsky, M., Positive forms on hyperkahler manifolds, arXiv:0801.1899, 33 pages.

Institute of Theoretical and Experimental Physics B. Cheremushininskaya, 25, Moscow, 117259, Russia

E-mail address: verbit@mccme.ru 